\title{
Prediction of ischaemic stroke in non-valvular atrial fibrillation if advanced echocardiography plays the game
}

\section{Patrizio Lancellotti ${ }^{1,2 *}$ and Maurizio Galderisi ${ }^{3}$}

\begin{abstract}
${ }^{1}$ University of Liège Hospital, Department of Cardiology and Cardiovascular Surgery, Heart Valve Clinic, and GIGA Cardiovascular Sciences, Centre Hospitalier de Liège du Sart Tilman, Liège, Belgium; ${ }^{2}$ Gruppo Villa Maria Care and Research, Anthea Hospital, Bari, Italy; and ${ }^{3}$ Department of Advanced Biomedical Sciences, Federico II University Hospital, Naples, Italy
\end{abstract}

Online publish-ahead-of-print 9 February 2018

\author{
This editorial refers to 'Left atrial function to identify \\ patients with atrial fibrillation at high risk of stroke: \\ new insights from a large registry' ${ }^{\dagger}$, by M.L. Leung et al., on \\ page 1416.
}

Atrial fibrillation (AF) presents a five-fold increase of the risk for ischaemic stroke and $20-30 \%$ of ischaemic strokes appear to be directly associated with AF. ${ }^{1}$ Currently, the need for anticoagulation for nonvalvular $A F$ is assessed on the basis of clinical parameters using the $\mathrm{CHAD}_{2} \mathrm{DS}_{2}$-VASc score. ${ }^{2}$ Conventional echocardiographic parameters such as left atrial (LA) dilatation, LA appendage thrombus > sludge $>$ smoke, and reduced LA appendage velocities $(<20 \mathrm{~cm} / \mathrm{s})$ can further risk stratify AF patients who would most benefit from anticoagulation. ${ }^{3}$ However, most of these parameters require transoesophageal echocardiography and do not evaluate the full risk spectrum related to LA functional impairment in AF. Moreover, whether they remain predictive of stroke after adjustment for comorbid factors (e.g. age, hypertension, diabetes, and previous stroke), which are the most critical upstream concomitant conditions promoting atrial remodelling, has not been well examined. Beyond the usual methods, including transmitral flow and changes in LA area and volume, novel techniques such as tissue Doppler imaging (TDI) and strain imaging provide more accurate estimation of LA function. LA strain is correlated with LA fibrosis in patients with $A F$, is significantly reduced in those with prior stroke, and predicts subsequent stroke. ${ }^{4}$ In their study, Leung et al. further extended this observation in a large registry cohort of 1361 patients with first diagnosis of $A F^{5}$. At baseline echocardiography, LA volumes, LA reservoir strain, $\mathrm{P}$-wave to $A^{\prime}$ duration (PA)-TDI (tissue Doppler imaging), and left ventricular (LV) global longitudinal strain were evaluated in patients with and without stroke. During follow-up (mean: 7.9 years), 100 patients (7\%) developed an ischaemic stroke, representing an annualized stroke rate of $0.9 \%$ (1.2\% in patients who had a $\mathrm{CHA}_{2} \mathrm{DS}_{2}$-VASc score of $\left.\leq 1\right)$. LA reservoir strain was reduced while PA-TDI was lengthened in the stroke compared with non-stroke group, and emerged as independently associated with risk of stroke in a model including $\mathrm{CHA}_{2} \mathrm{DS}_{2}-\mathrm{VASc}$ score, age, and anticoagulant use.

Notably, these two parameters fitting with the prediction of ischaemic stroke have a deep physiopathological meaning, as they are both an expression of LA function. PA-TDI represents LA electromechanical delay and can be obtained by calculating the time duration difference between the onset of the echocardiogram-derived P-wave and the peak of the A' wave on TDI. ${ }^{6}$ PA-TDI reflects the total LA activation time, and is likely a surrogate measurement of myocardial fibrosis and LA remodelling. ${ }^{6}$ PA-TDI has already been identified as an independent predictor of new-onset, ${ }^{7}$ post-operative, ${ }^{8}$ and recurrent AF after electrical cardioversion. ${ }^{9}$ Moreover, in a large population of 279 patients free of AF recurrence after successful catheter ablation, it was shown that the longer the LA electromechanical delay, the higher the risk of stroke. ${ }^{10}$ LA reservoir strain, i.e. the collection of flow from pulmonary veins during LV systole, can be quantified by two-dimensional speckle-tracking echocardiography as the peak positive longitudinal strain during LV systole. LA reservoir strain gives additional insights beyond traditional measures of $L A$ remodelling corresponding to LA enlargement, and its alteration is associated with a number of cardiac diseases including AF. ${ }^{11}$ In recent studies, LA reservoir strain has shown to provide incremental value for thromboembolic risk stratification over $\mathrm{CHAD}_{2} \mathrm{DS}_{2}-\mathrm{VASc}$ score, ${ }^{12}$ and has demonstrated a prognostic significance in various clinical settings. ${ }^{13}$ Interestingly, in the study of Leung et al., ${ }^{5}$ neither LA volume nor LA conduit strain - expression of LA flow passage to the left ventricle during early diastole- - and LV dimensions, LV ejection fraction, and global longitudinal strain predicted the risk of stroke. Of note, the relationship between LV systolic dysfunction and ischaemic stroke in AF is so evident that a reduced ejection fraction $(<40 \%)$ as

\footnotetext{
The opinions expressed in this article are not necessarily those of the Editors of the European Heart Journal or of the European Society of Cardiology.

† doi:10.1093/eurheartj/ehx736

* Corresponding author. Domaine Universitaire du Sart Tilman, Batiment B35, Department of Cardiology and Cardiovascular Surgery, University Hospital, Université de Liège, Centre Hospitalier de Liège du Sart Tilman, 4000 Liège, Belgium. Tel: +32 4366 71 94, Fax: +32-4366 71 95, Email: plancellotti@chu.ulg.ac.be

Published on behalf of the European Society of Cardiology. All rights reserved. (c) The Author(s) 2018. For permissions, please email: journals.permissions@oup.com.
} 
a surrogate of heart failure is already included in $\mathrm{CHA}_{2} \mathrm{DS}_{2}-\mathrm{VASc}$ risk score. $^{2}$

By identifying additional echocardiographic high-risk features, Leung et al. have elegantly refined the stroke risk assessment in patients with $A F^{5}$ The authors showed that adding advanced echocardiographic parameters, LA reservoir strain, and PA-TDI to the $\mathrm{CHA}_{2} \mathrm{DS}_{2}$-VASc risk stratification score improved the risk stratification of AF patients, and this may be useful to guide decisions regarding anticoagulation for patients upon first diagnosis of AF. It is known that AF patients with stroke risk factors have more severe ultrastructure abnormalities, which implies more serious LA remodelling and dysfunction with more stroke risk factors. ${ }^{4}$ Thus, higher $\mathrm{CHA}_{2} \mathrm{DS}_{2}$ VASc is intuitively associated with more significant enlargement LA, loss of atrial contraction, and reduced strain. Although, all this was confirmed in the study of Leung et al., the prediction of ischaemic stroke was observed independently of the $\mathrm{CHA}_{2} \mathrm{DS}_{2}-\mathrm{VASc}$ score. ${ }^{5}$ Both categories of patients at low and high risk of stroke were stratified in terms of stroke risk using LA reservoir strain and PA-TDI. In practice, although the $\mathrm{CHA}_{2} \mathrm{DS}_{2}-\mathrm{VASc}$ score provides a good indication of stroke risk based on common clinical risk factors, there remains the occurrence of stroke in patients with $\mathrm{CHA}_{2} \mathrm{DS}_{2}-\mathrm{VASc}$ score $\leq 1$ (a rate of $\leq 2.8 \%$ per year) in whom the European Society of Cardiology Guidelines do not routinely recommend antithrombotic therapy. ${ }^{2}$ In this group of patients, it is therefore desirable to provide personalized treatment according to tailored risk stratification, even if it is not well codified. The upcoming European Association of Cardiovascular Imaging AF Registry has been designed to provide additional insights into the relationships between LA structure and function with the development of ischaemic stroke and individual patient bleeding risk profiles. ${ }^{14}$

While the above-mentioned strengths must be emphasized, the only weakness of the study of Leung et al. ${ }^{5}$ corresponds to the use of advanced echocardiography itself as possible means of supporting $\mathrm{CHA}_{2} \mathrm{DS}_{2}-\mathrm{VASc}$ risk score in non-valvular $\mathrm{AF}$ patients. In particular, PA-TDI is a parameter that presupposes the use of colour TDI, which is angle-dependent and needs a high frame rate of recording. Therefore, its application in routine clinical practice has gradually been reduced. LA strain is a much more solid parameter, since it is obtainable on simple 2D images by speckle tracking echocardiography, which has high feasibility and good reproducibility. ${ }^{3,15}$ However, this technique is affected by a limited availability and the need for vendorspecific software on dedicated workstations for post hoc analyses, which cannot be obtained directly on the echocardiogram machine.

Because of its recognized value, echocardiography has become established in guidelines for the management of AF. ${ }^{2}$ In fact, echocardiography has a unique and important role in the assessment of cardiac structure and function, and risk stratification in AF patients. ${ }^{3}$ Altogether, the findings of Leung et $a l^{5}$ state the important concept that the assessment of $L A$ function adds valuable information to conventional clinical risk scores and improves the prediction of neurologic outcomes in patients with non-valvular AF. This novel approach has fundamental clinical implications and opens up new perspectives for the use of echocardiography as a support to the $\mathrm{CHA}_{2} \mathrm{DS}_{2}-\mathrm{VASc}$ risk score in $\mathrm{AF}$ patients with low-intermediate stroke risk. This could drive clinicians to make more accurate choices regarding oral anticoagulant therapy [oral anticoagulant $(\mathrm{OAC})$ ] in these delicate categories. The introduction of OAC may in fact be promoted in

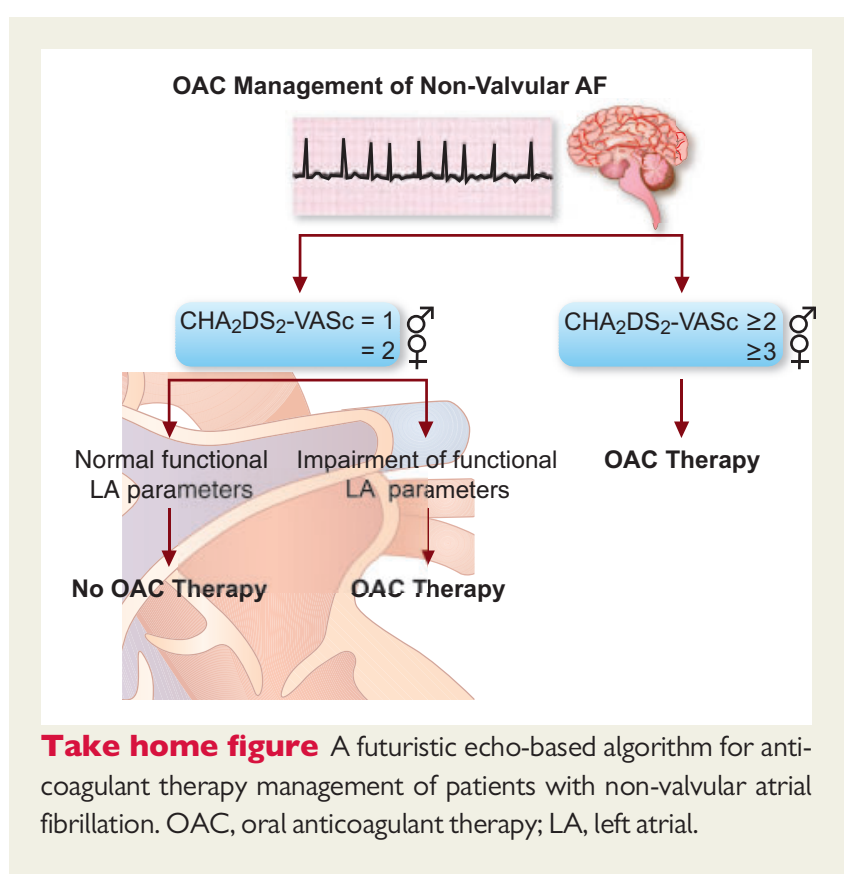

men with $\mathrm{CHA}_{2} \mathrm{DS}_{2}-\mathrm{VASc}=1$ and women with $\mathrm{CHA}_{2} \mathrm{DS}_{2}-\mathrm{VASc}=$ 2, when a functional LA impairment, detectable by the echocardiography, is well evident (Take home figure). Beyond LA enlargement, LA dysfunction can make AF patients more prone to the development of LA internal cavity thrombi and subsequent ischaemic stroke. Further prospective data are needed to confirm the incremental prognostic value of LA function assessment in patients considered at low-intermediate risk.

Conflict of interest: none declared.

\section{References}

1. Kishore A, Vail A, Majid A, Dawson J, Lees KR, Tyrrell PJ, Smith CJ. Detection of atrial fibrillation after ischemic stroke or transient ischemic attack: a systematic review and meta-analysis. Stroke 2014;45:520-526.

2. Kirchhof P, Benussi S, Kotecha D, Ahlsson A, Atar D, Casadei B. 2016 ESC guidelines for the management of atrial fibrillation developed in collaboration with EACTS. Eur Heart J 2016;37:2893-2962.

3. Donal E, Lip GY, Galderisi M, Goette A, Shah D, Marwan M, Lederlin M, Mondillo S, Edvardsen T, Sitges M, Grapsa J, Garbi M, Senior R, Gimelli A, Potpara TS, Van Gelder IC, Gorenek B, Mabo P, Lancellotti P, Kuck KH, Popescu BA, Hindricks G, Habib G, Cardim NM, Cosyns B, Delgado V, Haugaa KH, Muraru D, Nieman K, Boriani G, Cohen A. EACVI/EHRA Expert Consensus Document on the role of multi-modality imaging for the evaluation of patients with atrial fibrillation. Eur Heart J Cardiovasc Imaging 2016;17:355-383.

4. Azemi T, Rabdiya VM, Ayirala SR, McCullough LD, Silverman DI. Left atrial strain is reduced in patients with atrial fibrillation, stroke or TIA, and low risk CHADS (2) scores. J Am Soc Echocardiogr 2012;25:1327-1332.

5. Leung ML, van Rosendael PJ, Abou R, Ajmone Marsan N, Leung DY, Delgado V, Bax J. Left atrial function to identify patients with atrial fibrillation at high risk of stroke: new insights from a large registry. Eur Heart J 2018;39:1416-1425.

6. Muller P, Hars C, Schiedat F, Bosche LI, Gotzman M, Strauch J., Dietrich JW, Vogt JW, Tannapfel A, Deneke T, Mugge, Ewers A. Correlation between total atrial conduction time estimated via tissue Doppler imaging (PA-TDI Interval), structural atrial remodeling and new-onset of atrial fibrillation after cardiac surgery. J Cardiovasc Electrophysiol 2013;24:626-631.

7. De Vos CB, Weijs B, Crijns HJ, Cheriex EC, Palmans A, Habets J, Prins $M H$, Pisters R, Nieuwlaat R, Tieleman RG. Atrial tissue Doppler imaging for prediction of new-onset atrial fibrillation. Heart 2009;95:835-840.

8. Özlü MF, Erdem K, Kırış G, Demirhan A, Ayhan SS, Erdem A, Öztürk S, Tekelioğlu ÜY, Yazıcı M. Predictive value of total atrial conduction time measured with tissue Doppler imaging for postoperative atrial fibrillation after coronary artery bypass surgery. J Interv Card Electrophysiol 2013;37:27-33. 
9. Müller P, Schiedat F, Dietrich J-W, Shin DI, Kara K, Mügge A, Deneke T. Reverse atrial remodeling in patients who maintain sinus rhythm after electrical cardioversion: evidence derived from the measurement of total atrial conduction time assessed by PA-TDI interval. J Echocardiogr 2014;12:142-150.

10. Chao TF, Lin YJ, Tsao HM, Chang SL, Lo LW, Hu YF, Tuan TC, Li CH, Chang HY, Wu TJ, Yu WC, Chen SA. Prolonged atrium electromechanical interval is associated with stroke in patients with atrial fibrillation after catheter ablation. J Cardiovasc Electrophysiol 2013;24:375-380.

11. Cameli M, Mandoli GE, Loiacono F, Sparla S, lardino E, Mondillo S. Left atrial strain: A useful index in atrial fibrillation. Int J Cardiol 2016;220: 208-213.

12. Obokata M, Negishi K, Kurosawa K, Lo LW, Hu YF, Tuan TC, Li CH, Chang HY, Wu TJ, Yu WC, Chen SA. Left atrial strain provides incremental value for embolism risk stratification over $\mathrm{CHA}_{2} \mathrm{DS}_{2}$-VASc score and indicates prognostic impact in patients with atrial fibrillation. I Am Soc Echocardiogr 2014;27:709-716.e4.

13. Smiseth OA, Torp H, Opdahl A, Haugaa KH, Urheim S. Myocardial strain imaging: how useful is it in clinical decision making? Eur Heart J 2016;37:1196-1207.

14. Galderisi M, Donal E, Magne J, Lo ludice F, Agricola E, Sade EL, Cameli M, Schwammenthal E, Cardim N, Cosyns B, Hagendorff A, Neskovic AN Zamorano JL, Lancellotti P, Habib G, Edvardsen T, Popescu BA. Rationale and design of EACVI AFib Echo Europe Registry for assessing relationships of echocardiographic parameters with clinical thromboembolic and bleeding risk profile in non-valvular atrial fibrillation. Eur Heart J Cardiovasc Imaging 2018; in press.

15. Rosca M, Lancellotti P, Popescu BA, Piérard LA. Left atrial function: pathophysiology, echocardiographic assessment, and clinical applications. Heart 2011;97 1982-1989. 